\title{
Correction to: Cloning and characterization of a monoterpene synthase gene from flowers of Camelina sativa
}

\author{
Monica Borghi $^{1,2} \cdot$ De-Yu Xie ${ }^{1}$
}

Published online: 13 November 2017

(C) Springer-Verlag GmbH Germany, part of Springer Nature 2017

\section{Correction to: Planta https://doi.org/10.1007/ s00425-017-2801-x}

In the original publication, the order of figures and citations was incorrect. The corrections are listed below:

Page 2, left panel, line 17:

In the following sentence "codified" should be replaced by "codifying" and "has" should be replaced with "have". The sentence should read:

TPS genes have been isolated from many plant species, their codifying proteins have been biochemically...

Page 2, right panel, last line:

In the following sentence "database" is redundant and should be removed. The final sentence should read:

...searches against the transcripts of Arabidopsis (TAIR $10)$.

Page 3, left panel, second paragraph, first line:

"namely-CSTPSI": space "-" should be added between "namely and -". The final sentence should read: The fulllength sequence of CSTPS1 and a shorter version (namely -CsTPS1) lacking...

Page 3, left panel, second paragraph:

The online version of the original article can be found under https://doi.org/10.1007/s00425-017-2801-x.

De-Yu Xie

dxie@ncsu.edu

Monica Borghi

MBorghi@ mpimp-golm.mpg.de

1 Department of Plant and Microbial Biology, North Carolina State University, Raleigh, NC 27695, USA

2 Present Address: Max-Planck-Institute of Molecular Plant Physiology, Am Mühlenberg, 1, 14476 Potsdam-Golm, Germany
In the following sentence the preposition "with" is repeated twice. Please remove one of the two prepositions. The final sentence should read:

LB solidified with agar supplemented with antibiotics...

Page 4, left panel, line 12 from the top:

"Agrobacterium" should be written with normal font and not in Italic. The final sentence should read:

Overnight-grown Agrobacterium cells were used for the transformation...

Page 4, right panel, line 8 from the top:

"Agrobacterium" should be written with normal font and should not be in Italic. The final sentence should read:

Both constructs were transformed into Agrobacterium as previously described

Page 5, Figure caption of Fig. 1:

"chloroplast transit peptide" should be replaced with "plastid targeting peptide (PTP)". The final sentence should read:

The amino acids sequence underlined in black shows the plastid targeting peptide (PTP).

Page 6, left panel, line 7 from the top

A reference to Fig. 2 (current Fig. 7) should be reported here. The final sentence should read:

... and minor amounts of limonene $(4.7 \%)$ and $\alpha$-tepinolene (4.7\%, Fig. 2).

Page 6, left panel, line 10 from the top:

Fig. 2 should be renamed as Fig. 3 and the final sentence should read:

VOCs collection from wild-type Camelina flowers revealed emission of multiple terpene compounds (Fig. 3).

Page 6, right panel, line 14 from the top:

Fig. 3 should be renamed as Fig. 4. The final sentence should read:

As shown in Fig. 4, the X-Glu solution conferred a blue hue 
Page 6, right panel, line 18 from the top:

Fig. 3 should be renamed as Fig. 4. The final sentence should read:

...found in root in correspondence of the points of lateral root emergence (Fig. 4).

Page 6, right panel, line 21 from the top:

Fig. 4 should be renamed as Fig. 5. The final sentence should read:

...detected in roots and leaves (Fig. 5), which confirmed the...

Page 6, right panel, line 9 from the beginning of the second paragraph:

Fig. 5 should be Fig. 6. The final sentence should read:

... chlorophylls in chloroplasts (Fig. 6, panels on the left).

Page 6, Figure caption:

Fig. 2 should be renamed as Fig. 3. The final sentence should read:

Fig. 3 Total ion chromatogram

Page 7, Figure caption:

Fig. 3 should Fig. 4. The final sentence should read:

Fig. 4 Activation pattern of CSTPS1 promoter.

Page 7, right panel, fifth line from the bottom of the page:

Fig. 6 should be renamed as Fig. 7. The final sentence should read:

modifying enzymes (P450 monooxygenases) was observed (Fig. 7).

Page 8, Figure caption:

Fig. 5 should Fig. 6. The final sentence should read:

Fig. 6 Transient expression of reporter proteins in chloroplasts of Nicotiana tabacum (tobacco).

Page 8, left panel, third line:

Fig. 2 should be renamed as Fig. 3. The final sentence should read:

Camelina flowers release a bouquet of scented VOCs, which include monoterpenes, sesquiterpenes, and sulfurcontaining compounds (Fig. 3).
Page 8, left panel, line 10:

Fig. 7 should be renamed as Fig. 2. The final sentence should read:

...limonene, $(E)$ - $\beta$-ocimene, $(Z)$ - $\beta$-ocimene and $\alpha$-terpinolene (Fig. 2).

Page 8, right panel, line 5:

Fig. 5 should be renamed as Fig. 6. The final sentence should read:

...the cytosol to plastids (Fig. 6). After the genome sequence of...

Page 10, right panel, line 4 from the bottom of the page:

Several should be removed. The final sentence should read:

Camelina has been the target of numerous...

Page 11, figure caption:

Fig. 6 should be renamed as Fig. 7. The final sentence should read:

Fig. 7 Localization of TPS genes in the genome of Camelina. The...

Page 12, figure caption:

Fig. 7 should be renamed as Fig. 2. The final sentence should read:

Fig. 2 Total ion chromatogram (TIC) and spectra of terpene produced...

Page 13, left panel, line 32 from the top of the page:

Fig. 6 should be renamed as Fig. 7. The final sentence should read:

These clusters are exclusively composed of TPS genes or may include prenyltransferase genes and terpene modifying enzymes such as P450 genes (Fig. 7).

The original article was corrected. 